\title{
New Compact Microstrip Filters Based on Quasi Fractal Resonator
}

\author{
Yaqeen S. Mezaal ${ }^{1}$, Hiba H. Saleh ${ }^{2}$ and Hussam Al-saedi ${ }^{3}$ \\ ${ }^{1}$ Medical Instrumentation Engineering Department, Al- Esraa University College, Baghdad, Iraq \\ 2 Department of Electrical Engineering, University of Turkish Aeronautical Association, Ankara, Turkey \\ ${ }^{3}$ Department of Electrical and Computer Engineering, University of Waterloo, Waterloo, Canada \\ Corresponding Author: yakeen_sbah@yahoo.com
}

\begin{abstract}
This paper presents new microstrip devices as single band bandpass and multi band bandstop filters. The proposed filters use slotted patch microstrip resonator based on quasi fractal geometry, simulated by AWR12 software package. Both filters have quasi elliptic frequency responses, designed at centre frequency of $2.437 \mathrm{GHz}$ for bandpass filter and at band frequencies of 2.434, 4.032, 4.976 and $5.638 \mathrm{GHz} \mathrm{GHz}$ respectively, for multi band bandstop filter. All filters are employed using RT/Duroid 6010.8 LM substrate of 10.8 dielectric constant and 1.27 $\mathrm{mm}$ dielectric thickness. Simulation results show that the designed quasi fractal bandpass filter has very narrow fractional bandwidth of $0.38 \%$ which is very rare in microstrip filter design. On the other hand, the projected bandstop filter offers multi narrow rejection bands that is useful in broadband wireless schemes influencing from fixed interferences. Both filters present satisfactory S11 and S21 responses besides smallness properties that stand for interesting features of the newest wireless applications. The simulated and measured frequency responses for both designed filters are in good agreement.
\end{abstract}

\section{Introduction}

The expansion of signal processing algorithms and hardware devices is essential to sustain the swift development of the latest and complicated wireless services. Particularly, contemporary microwave architectures should satisfy progressively rigorous requirements regarding their performances, compactness and easy integration within other devices or systems [1]. Microstrip bandpass filter (BPF) and bandstop filter (BSF) are electromagnetic elements which are situated at the terminal and beginning of transmitter and receiver respectively for wireless communication systems. These devices represent two port networks that are important to provide signal transmission and rejection within specific bands according to wireless system requirements[2]. The elemental part of these devices is called resonator. The topology of resonators can be categorized into planar resonators and non-planar resonators. The planar resonators are regularly constructed using the microstrip technology. Microstrip resonators are extremely planar resonators and have huge possibilities to diminish the filter size. Meandering the microstrip resonator is the most adopted methods to miniaturize the microstrip filter. For instance, the size of open loop meandered microstrip line resonator is less than $\lambda_{g 0} / 8$ by $\lambda_{g 0} / 8$, where $\lambda_{g 0}$ is the guided wavelength at fundamental frequency [3]. Nevertheless, the microstrip line must have too narrow line if more smallness using meandering is intended. This case will decrease the quality factor of the resonator as the line becomes narrower. This problem has been fixed by High Temperature Superconducting (HTS) materials. HTS technology has been widely used to design very narrow fractional bandwidth filters of less than $1 \%$ with huge external quality factor that is very difficult to be realized in the case of classical microstrip filters. However, the cooling requirements lessen the compactness gain. In contrast, non-planar resonators have moderately better quality factor as compared with planar resonators, but they are larger in size as in waveguide cavity filters. These nonplanar filters can be utilized in wireless systems when the frequency responses of these devices are more essential than the filter size as in the applications of ground cellular stations and satellite transponders[4].

The speedy growth of military and marketable applications has motivated new requirements of analog filters in terms of low profile and compactness. One of the smart solutions for all these demands is the use of fractal geometry $[5,6]$.

The fractal idiom is initiated from the Latin word "fractus," which stands for split or broken parts. Benoit Mandelbrot is a mathematician who firstly defined the fractal and explained all its curves, iterations and length specifications. Fractals are constructed using a particular formula with different curve orders which is called Iterated Function Systems (IFS). They are created from 
the sum of copies of itself; each copy has smaller copies from the previous orders [7].

Along with the initial predictions of the use of fractals in the design and fabrications of filters is that of Yordanov et. al, [8]. They had designed and fabricated fractal filter. Their investigations are based on Cantor fractal geometry. Microstrip fractal filters, subsequently, had been studied widely in the literature using various fractal geometries such as Hilbert, Minkowski, Koch, Peano and Moore curves. These geometries are productively used in the design of compact single band and dual/ multi band microstrip filters for various communication applications [9-17].

Hilbert and Minkowiski fractal geometries had been used to model and fabricate very compact HTS filters as reported in [9]. The designed filters are in diverse fractal iteration levels for different wireless applications including ISM band systems. Also, a number of $4^{\text {th }}$ order topologies, with Chebychev and quasi elliptic responses, had been manufactured. Minkowski-like pre-fractal resonators are used to design microstrip BPFs as presented in [10] for ISM band applications. These filters use dual-mode fractal resonator as $2^{\text {nd }}$ order EM component in single structure with orthogonal $\mathrm{I} / \mathrm{O}$ feeders and perturbation element. Microstrip BPFs using Hilbert fractal resonators with coupling stubs have been designed for ISM band wireless application as reported in [11]. These filters have narrow band frequency responses with dual transmission zeros as rejection band levels. The fractal resonators are based on $2^{\text {nd }}$ and $3^{\text {rd }}$ iteration levels. A study on dual-mode BPFs based on Koch-like prefractal fractal geometries with multiple iteration structures for ISM band applications at $2.4 \mathrm{GHz}$ has been presented in [12]. Koch resonators for these BPFs have been used with comer cross slotted perturbation and cut perturbation techniques. Frequency responses for these filters explained that the designed BPFs have quasi elliptic and Chebyshev performance curves. A perceptible size reduction is noticed as the iteration order increases at the same frequency and using the same substrate material. Peano fractal resonators had been adopted to design microstrip filters as single and dualmode circuits [13,14,15]. A compact microstrip BPF has been presented in [13] as four pole filter. It has four capacitively coupled resonators using $2^{\text {nd }}$ iteration level of Peano fractal strips. Peano fractal microstrip BPF with second harmonic suppression has been reported in [14]. In this study, two single-mode capacitively coupled resonators comprise the filter configuration. Dual-mode resonator based on Peano fractal geometry had been employed to simulate a condensed microstrip BPF with a quasi-elliptic frequency response at $2.45 \mathrm{GHz}$ [15]. This filter has been constructed by using four parts, each with a constitution of $2^{\text {nd }}$ iteration level of Peano geometry. Moore fractal BPFs as two-pole configurations had been designed in [16] for ISM band wireless system. These filters employ two edge coupled resonators based on $2^{\text {nd }}$ and $3^{\text {rd }}$ iteration levels of Moore fractal geometries. Both filters have very good electrical specifications and they have superior responses than Hilbert fractal BPFs at same designed frequency and substrate specification as well as their noticeable miniaturization and $2^{\text {nd }}$ harmonic suppression. New microstrip BPF and BSF based on Hilbert fractal geometry has been presented in [17].The designed BPF uses dual edge coupled Hilbert resonators based on $2^{\text {nd }}$ iteration level and it has wide band frequency response designed at 2 and $2.2 \mathrm{GHz}$ resonant frequencies. On the other hand, BSF has been designed using dual directly coupled Hilbert resonators of the same iteration level and substrate specifications at $2.37 \mathrm{GHz}$ centre frequency. This BSF filter has narrow rejection bandwidth which can be used in broadband wireless schemes that are susceptible to fixed frequency interferences. New dualmode microstrip BPF has been designed around $2 \mathrm{GHz}$ as stated in [18]. It uses slotted patch resonator based on customized Cantor fractal geometry. The S11 and S21 frequency responses are interesting and parametric investigation about the effect of perturbation length on the filter electrical specifications has been achieved in this study.

There are some constraints of microwave fractal devices including antennas and filters in terms of electrical specifications [9]. Nevertheless, these constraints can be fixed by reformation techniques as in fractal reconfiguration [19-21] and like pre-fractal geometries $[10,12,22-23]$. All of them can be called semi or quasifractals [19], that can be applied to filter design, but not a precisely fractal geometry with unlimited scale. Thus, a quasi- fractal with limited iterations can be adopted for an exacting frequency band. Each is related to a definite scale of the fractal structure.

In this research article, condensed microstrip filters based on dual-mode resonator have been designed as bandpass and bandstop filters. They use quasi fractal resonator as slotted patch EM elements. The presented quasi fractal BPF has been designed at $2.437 \mathrm{GHz}$ for ISM band wireless application with hugely narrow fractional bandwidth of $0.38 \%$. On the other hand, quasi fractal BSF has been designed for multi narrow rejection band frequency responses that can be employed in broadband wireless systems sensitive to fixed frequency interferences for multi service applications of ISM and C band wireless systems. Both microstrip filters have interesting compactness and satisfactory simulated S11 and S21 responses that are well matched with the measurements. 


\section{Filter Design}

The essential theory for a microstrip filter modelling is the required properties of the whole categories of resonant modes. At present, microstrip filters design mostly focus on dual-mode (doubly tuned circuits) structures. Nevertheless, the upper modes don't have enough practical realizations. Currently, patch and loop microstrip resonators $[18,26]$ in different forms, hold the vast attention of microwave circuit manufacturers for fabricating new microwave devices and enhancing the frequency responses. A microstrip doubly tuned resonator classically has planar or two-dimensional (2-D) symmetry for different topologies. Figure 1 illustrates some instances of microstrip doubly tuned resonator, where $D$ on the top of all resonators in this illustration points to its regular dimension, and $\lambda_{g o}$ stands for the guidedwavelength at its specified resonance. A miniature patch or slit as induction and coupling element has been placed to each resonator at $45^{\circ}$ from their $\mathrm{x}$-axis for each structure as shown in Figure 1.

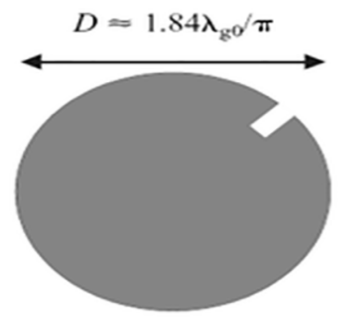

(a)

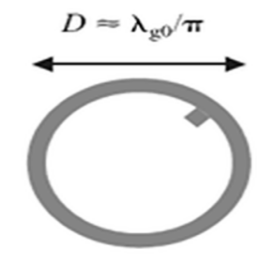

(c)

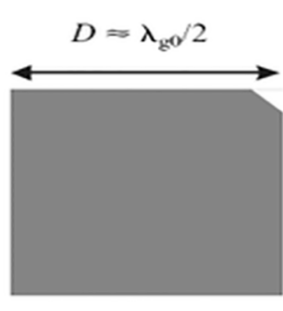

(b)

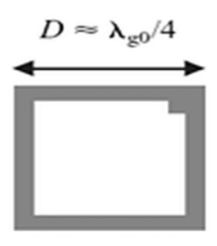

(d)

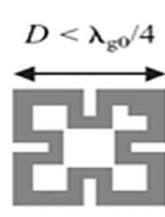

(e)
Figure 1. Dual-mode microstrip resonators [1]

The proposed filters have been simulated by Advanced Wave Research version 12 (AWR12) simulator. This electromagnetic simulator has been customized to use RT/Duroid 6010.8 LM substrate of 10.8 dielectric constant, $1.27 \mathrm{~mm}$ dielectric thickness and $35 \mu \mathrm{m}$ conductor thickness. The guided wave length $\left(\lambda_{g o}\right)$ can be evaluated by [1] :

$$
\lambda_{g o}=\frac{\mathrm{c}}{\mathrm{f}_{0} \sqrt{\varepsilon_{\text {eff }}}}
$$

Where $c$ stands for light speed, $f_{0}$ is center frequency and $\varepsilon_{e f f}$ is effective dielectric constant that can be determined roughly from [17]:

$$
\varepsilon_{\text {eff }}=\frac{\varepsilon_{r}+1}{2}
$$

Where $\varepsilon_{r}$ stands for relative substrate constant. Eq.(1) is very helpful to correspond to the size of microstrip filter in terms of $\lambda_{g o}$ in order to compare clearly the smallness of diverse filter topologies irrespective of their operating frequency, type of substrate material and external dimensions.

In this study, the filters have been considered as quasi fractal devices since the used resonator has self-similarity and uniform slots like fractals as well as the topology of resonator is newly investigated in this study that let us to refer it as quasi-fractal resonator. The nature of quasi fractal resonator stands for active perturbation effect to the electromagnetic equilibrium of the resonator configuration. Hence, the electromagnetic distributions of the degenerated modes are going to be no longer orthogonal and combined to each other.

Two orthogonal feeds in the input and output sides (I/O feeds) of quasi fractal resonator have been used in the BPF design. On the other hand, I/O straight line feed has been adopted in the BSF design of this study. All feeds have 50 Ohm impedance as standard in filter construction.

The majority of the EM simulators are rooted from the method of moment as an evaluation procedure. They determine the frequency responses of filter by primary partitioning the resonators in diminutive mesh, fewer or further fixed in accordance with the considered precision, and subsequently resolving linear integral equations. It is essential to describe the electromagnetic coupling of quasi fractal resonator with the feed circuit. This can be done by evaluating external quality factor $\left(Q_{e x t}\right)$. The resonator can be singly loaded resonator by just coupling to input port or to be doubly loaded resonator if it is connected to both input/output ports $[1,9]$. For singly loaded resonator, $Q_{e x t}$ can be determined from the phase response of $S_{11}$ as illustrated in Figure 2 by:

$$
Q_{\text {ext }}=\frac{f_{\left(0^{\circ}\right)}}{f_{\left(-90^{\circ}\right)}-f_{\left(+90^{\circ}\right)}}
$$

While in the case of doubly loaded resonator as in in our study, $Q_{\text {ext }}$ can be derived by definition from equivalent 
$\left|S_{21}\right|$ transmission response within $3 \mathrm{~dB}$ band frequencies of $f_{1}$ and $f_{2}$ symmetrically around $f_{0}[9]$ :

$$
Q_{e x t}=\frac{2 f_{o}}{f_{2}-f_{1}}=\frac{2 f_{o}}{B W_{3 d B}}
$$

Where $f_{o}$ is the center frequency and $B W_{3 d B}$ represents $3 \mathrm{~dB}$ bandwidth transmission peak of $\mathrm{S} 21$ (or S12) filter frequency response.

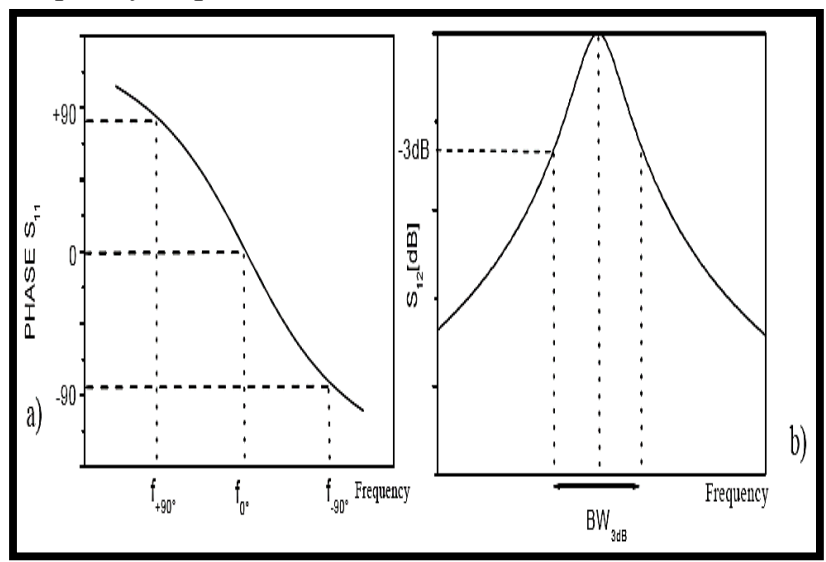

Figure 2. $Q_{\text {ext }}$ computation for (a) Singly and (b) Doubly loaded resonator

The modeled quasi fractal BPF is illustrated in Figure 3. It has been constructed using dual-mode patch resonator with regular slots and perturbation element representing by small square patch on the right up corner of quasi fractal resonator. Consequently, this microstrip filter represents two-pole topologies in single structure due to degenerated electromagnetic modes of employed resonator. The perturbation square patch side length $(\mathrm{k})$ is $1 \mathrm{~mm}$, while the spacing ( $\mathrm{s}$ ) among slotted patch resonator and $\mathrm{I} / \mathrm{O}$ feeds is $0.4 \mathrm{~mm}$ and $\mathrm{I} / \mathrm{O}$ feeder width $(\mathrm{t})$ is $1 \mathrm{~mm}$. The length of $\mathrm{r}$ that can be used easily to generate quasi fractal resonator is $2 \mathrm{~mm}$. Consequently, the external length of quasi fractal resonator as slotted patch topology is $18 \mathrm{~mm}$. The overall substrate size in this designed BPF is $24 \times 24 \mathrm{~mm}^{2}$. By using the same quasi fractal resonator, multi band BSF has been designed as shown in Figure 4 using the same external dimensions, coupling gap and substrate specifications but with using straight line I/O feed of 24 mm length.

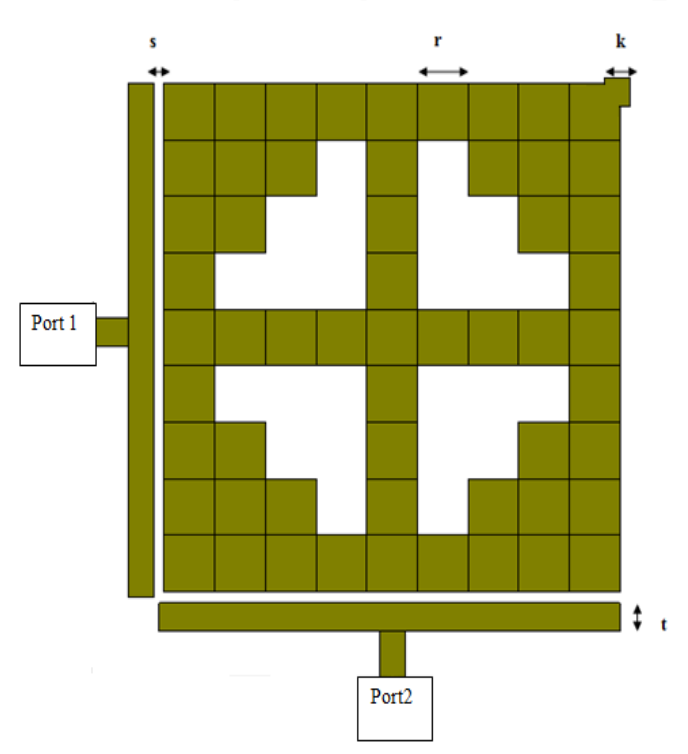

Figure 3. Quasi fractal dual-mode BPF

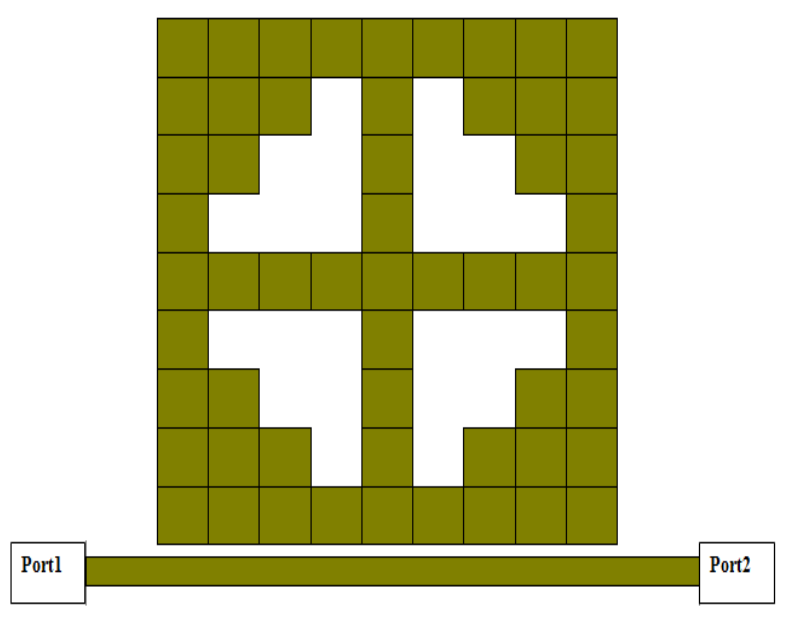

Figure 4. Quasi fractal multi band BSF

\section{Performance Evaluation}

Dual degenerate mode microstrip BPF employing quasi fractal resonator is simulated by AWR12 software package. The frequency response for depicted quasi fractal BPF in Figure 3 is shown in Figure 5 . The return loss and insertion loss values are 12.426 and $0.2416 \mathrm{~dB}$ respectively at centre frequency of $2.437 \mathrm{GHz}$. Dual transmission poles at 2.435 and $2.4392 \mathrm{GHz}$ in the $3 \mathrm{~dB}$ passband region can be detected noticeably. It is apparent from this graph that there are two transmission zeros correspond to band stop levels at 2.393 and $2.534 \mathrm{GHz}$ and they can be observed distinctly with attenuation poles of 
98.518 and $97.122 \mathrm{~dB}$. Regarding S12 response, it has the same S21 response since the topology of filter has self similarity. This filter has very narrow bandwidth of 9.2 $\mathrm{MHz}$ which is usually a big goal in wireless systems to cause the filter able to prevent the interfering signals running in the neighbouring bands.

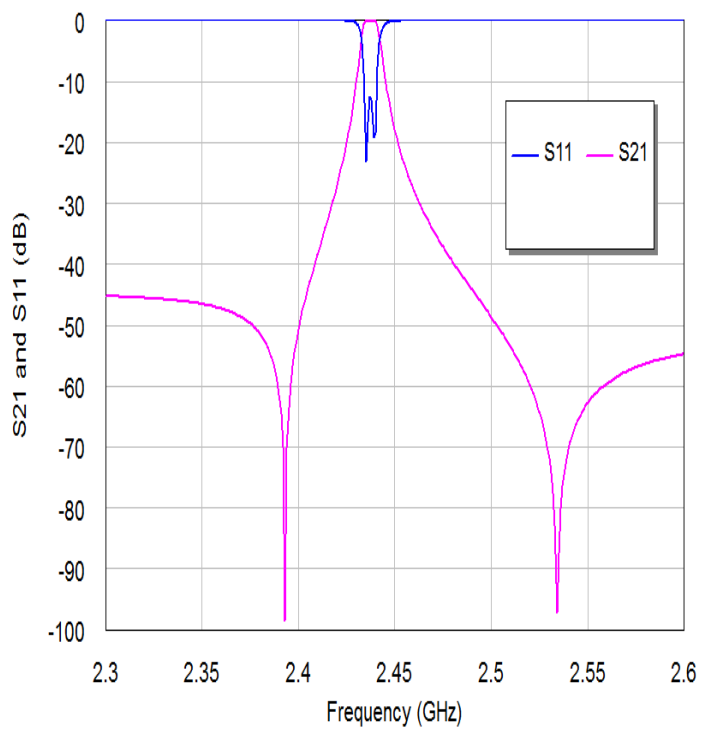

Figure 5. The frequency responses of the microstrip quasi fractal bandpass filter

The quasi fractal BSF is depicted in Figure 4. The uniform slot of adopted resonator with straight line $\mathrm{I} / \mathrm{O}$ feeder have EM effects for filter structure to provide multi stop band responses at 2.434, 4.032, 4.976 and $5.638 \mathrm{GHz}$ respectively, within 2 to $6 \mathrm{GHz}$ sweeping frequency range as depicted in Figure. 6. The electrical specifications for first band are $1.9818 \mathrm{~dB}$ return loss, 23.466 insertion loss and $33.1 \mathrm{MHz}$ bandwidth. For the second band, the frequency response has $0.0105 \mathrm{~dB}$ return loss, $42.775 \mathrm{~dB}$ insertion loss and $31.7 \mathrm{MHz}$ rejection bandwidth. The magnitudes of return loss, insertion loss and rejection bandwidth for third band are $0.081747 \mathrm{~dB}, 17.294 \mathrm{~dB}$ and 4.1 MHz respectively. Lastly, for the fourth band, the magnitudes of return loss, insertion loss and rejection bandwidth are $1.4067 \mathrm{~dB}, 30.631 \mathrm{~dB}$ and $43.6 \mathrm{MHz}$ respectively. This filter is very advantageous in broadband wireless schemes which are susceptible to static frequency interferences for multi service applications of ISM and $\mathrm{C}$ band wireless systems.

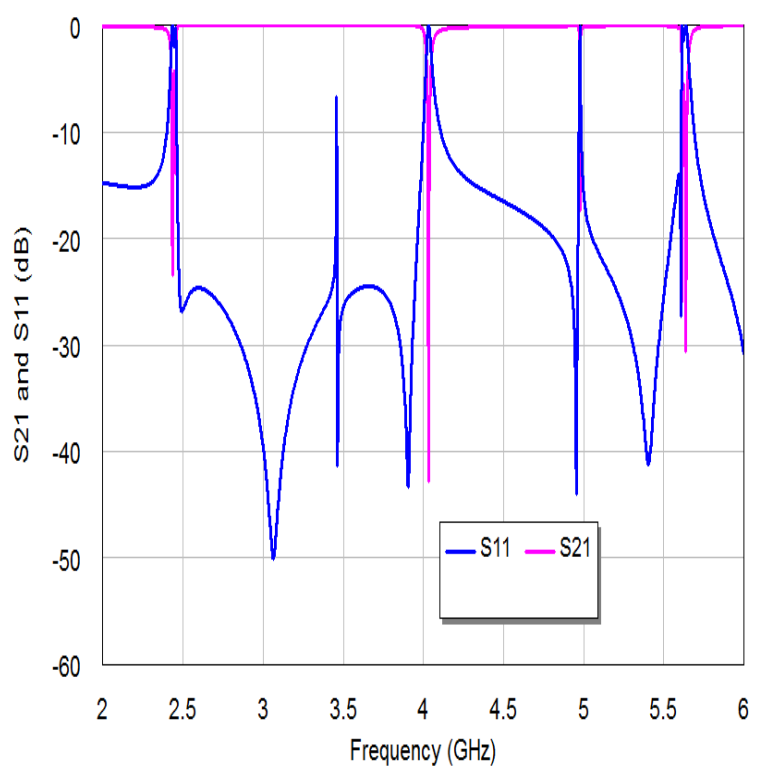

Figure 6 . The frequency response of multi bandstop quasi fractal filter

Figures 7-8 give an idea about the scattering phase responses of the modelled filters for S11 and S21 parameters respectively. Both of them have good level of phase response linearity which is the feature of quasi elliptic filters [17]. The phase response of quasi fractal BSF has higher jumping levels than quasi fractal BPF.

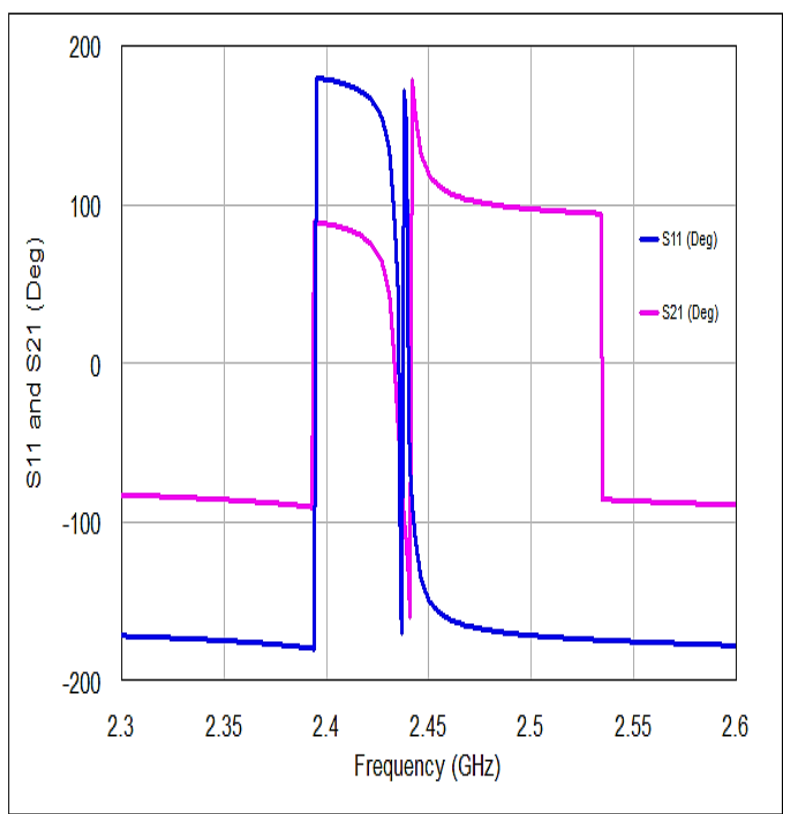

Figure 7. S21 and S11 phase responses of quasi fractal bandpass filter 


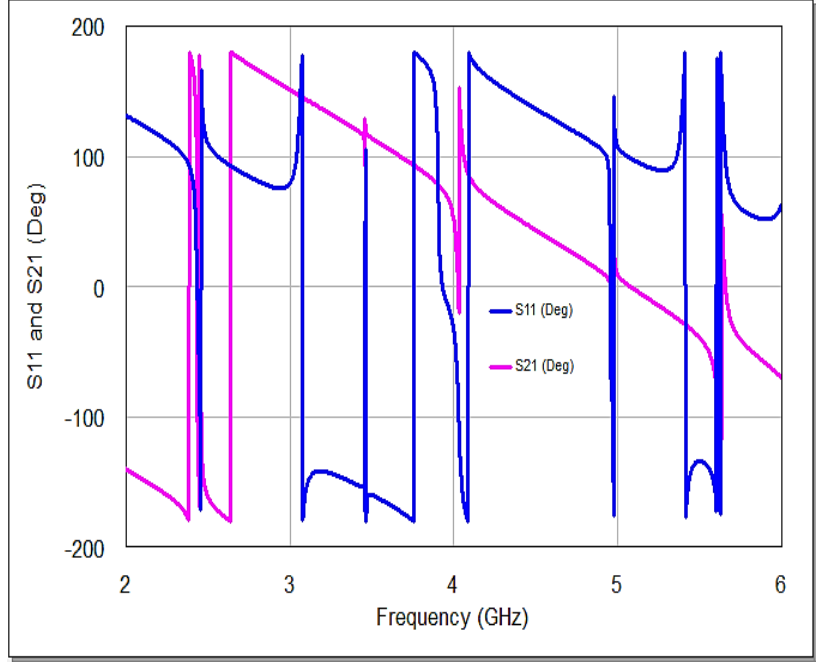

Figure 8. S21 and S11 phase responses of quasi fractal multi band BSF

The surface current density simulation results at 2.437 $\mathrm{GHz}$ for quasi fractal BPF and $2.434 \mathrm{GHz}$ for quasi fractal BSF, have been demonstrated in Figures 9 and 10 respectively which take the shape of adopted quasi fractal resonator. These responses are achieved using Sonnet EM simulator. In these figures, the red colour is a sign of the upper limit of coupling, whereas the blue colour explains the minimum one. The largest values of surface current intensity are visible in quasi BPF structure with magnetic intensity of $18 \mathrm{Amp} /$ Meter that are distributed in the up, middle and bottom arms of designed quasi fractal BPF. On the other hand, the maximum current intensity for quasi fractal BSF is $7 \mathrm{Amp} /$ Meter which are distributed mostly in the straight $\mathrm{I} / \mathrm{O}$ feed of BSF and corner sides of resonator.
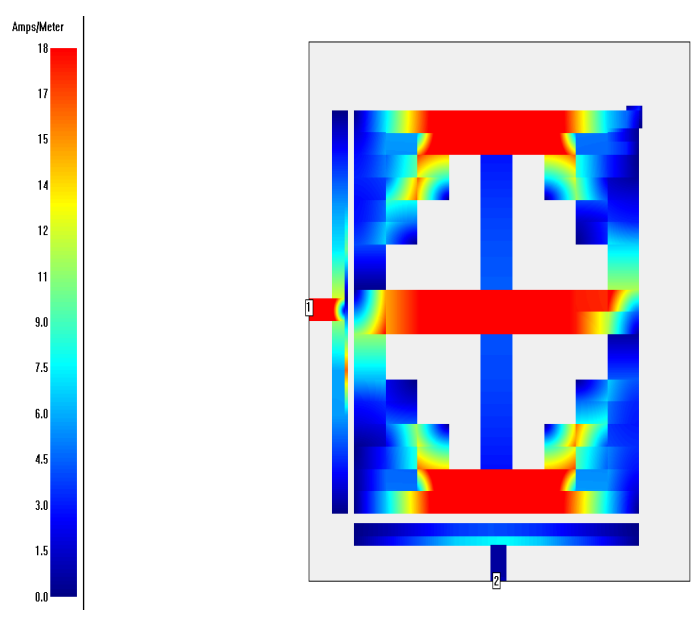

Figure 9. Current intensity distributions of quasi BPF at $2.437 \mathrm{GHz}$

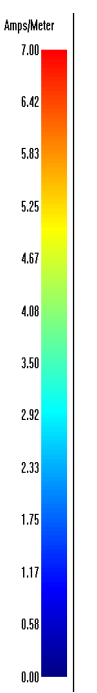

Figure 10 Current intensity distributions of quasi $\mathrm{BSF}$ at $2.434 \mathrm{GHz}$

In addition to considerable compactness, the proposed quasi fractal BPF in this paper has the narrowest fractional bandwidth, the best external quality factor and totally or almost better electrical filter specifications in terms of return loss and insertion loss than reported filters in [12, 27-32] used for ISM band wireless applications as illustrated in Table 1. Theoretically, very narrow microstrip BPF that has fractional bandwidth less than $1 \%$ is very rare, but in HTS filters is possible [24]. However, HTS filters require efficient coolers to decrease the resultant heat due to narrow strips of HTS topology. This, of course, decreases the compactness benefit of this type of filters. The proposed microstrip BPF in this study has interesting compactness and very narrow passband response of $0.38 \%$ fractional bandwidth that is very purposeful in wireless systems in excluding interfering signals in adjacent bands and increases hugely the external quality factor of designed filter. On the other hand, the designed bandstop filter has multi narrow reject band peaks that can be useful for wireless system applications that require wide passband responses with narrow reject band separations as in similar bandstop filter applications reported in $[17,25]$. The substrate size of quasi fractal BSF is about $0.473 \lambda_{g o} \times 0.473 \lambda_{g o}$, that makes the designed BSF in this study has higher compactness than reported multi bandstop filters in [25] operating at almost the same fundamental frequency. 
Table 1 Comparison of the designed dual-mode BPF with [12, 27-32] for ISM band application

\begin{tabular}{|c|c|c|c|c|c|c|c|c|}
\hline Filter Parameters & $\begin{array}{l}12] \text { for } 1^{\text {st }} \\
\text { iteration } \\
\text { structure }\end{array}$ & {$[27]$} & [28] & [29] & {$[30]$} & {$[31]$} & {$[32]$} & $\begin{array}{c}\text { Dual mode } \\
\text { BPF in this } \\
\text { study }\end{array}$ \\
\hline Center Frequency $(\mathrm{GHz})$ & 2.4 & 2.4 & 2.4 & 2.4 & 2.44 & 2.44 & 2.44 & 2.437 \\
\hline Insertion Loss(dB),S21 & 0.901 & 0.3 & 8.15 & 1 & 1.25 & 2.5 & 3 & 0.2416 \\
\hline Return Loss(dB),S11 & 7.25 & 16.5 & 35 & 20 & 25 & 15 & 15 & 12.426 \\
\hline $\begin{array}{c}\text { Dielectric Constant and } \\
\text { Thickness }\end{array}$ & $10.8,1.27 \mathrm{~mm}$ & $2.2,31 \mathrm{mil}$ & $4.4,1.6 \mathrm{~mm}$ & $3.55,0.813$ & $2.55,1 \mathrm{~mm}$ & $\begin{array}{c}4.41 .1 .6 \\
\mathrm{~mm}\end{array}$ & $\begin{array}{c}3.38,0.508 \\
\mathrm{~mm}\end{array}$ & $\begin{array}{c}10.8,1.27 \\
\mathrm{~mm}\end{array}$ \\
\hline Size $\left(\mathrm{mm}^{2}\right)$ & $\begin{array}{c}23.214 \times \\
23.214\end{array}$ & $58 \times 58$ & $23 \times 12$ & $57 \times 57$ & ............... & ............... & $17 \times 9$ & $24 \times 24$ \\
\hline Size by $\lambda_{\text {go }}{ }^{2}$ & $\begin{array}{c}0.451 \lambda_{g o} \mathrm{x} \\
0451 \lambda_{g o}\end{array}$ & $\begin{array}{c}0.587 \lambda_{g o} \mathrm{x} \\
0.587 \lambda_{g o}\end{array}$ & $\begin{array}{c}0.302 \lambda_{g o} \mathrm{x} \\
0.158 \lambda_{g o}\end{array}$ & $\begin{array}{c}0.688 \lambda_{g o} \mathrm{x} \\
0.888 \lambda_{g o}\end{array}$ & ….......... & …............. & $\begin{array}{l}0.2 \lambda_{g o} \mathrm{x} \\
0.08 \lambda_{g o}\end{array}$ & $\begin{array}{c}0.474 \lambda_{g o} \mathrm{x} \\
0.474 \lambda_{g o}\end{array}$ \\
\hline Bandwidth(MHz) & 30 & 25 & 130 & 1400 & 200 & 146.4 & 80 & 9.2 \\
\hline Fractional Bandwith (\%) & 1.25 & 1.04 & 5.42 & 58.33 & 8.2 & 6 & 3.28 & 0.38 \\
\hline $\begin{array}{l}\text { External Quality Factor } \\
\text { (Qext) }\end{array}$ & 160 & 192 & 36.92 & 3.43 & 24.4 & 3.33 & 61 & 530.43 \\
\hline
\end{tabular}

\section{Measurements}

Figures 11 and 13 show the prototype photographs of quasi fractal BPF and BSF. Quasi fractal resonator are printed on RT/Duroid 6010.8 LM substrate using the standard mask etching technique. Figures 12 and 14 illustrate the experimental and simulated frequency responses of these fabricated filters. The measurements of these prototypes are prepared using HP8720C vector network analyser using $50 \mathrm{Ohm}$ feeds. Both simulations and measurements for all designed filters in this study are in good agreement with slight acceptable differences. These differences are attributed to fabrication tolerances, simulation precision and connector soldering.

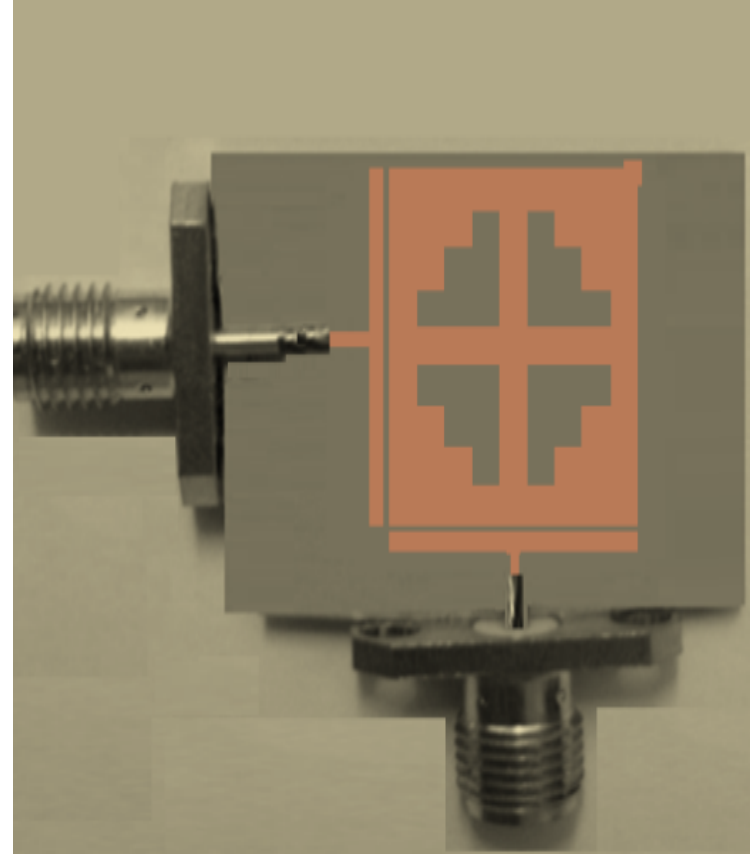

Figure 11. Photograph of the fabricated quasi fractal BPF. 


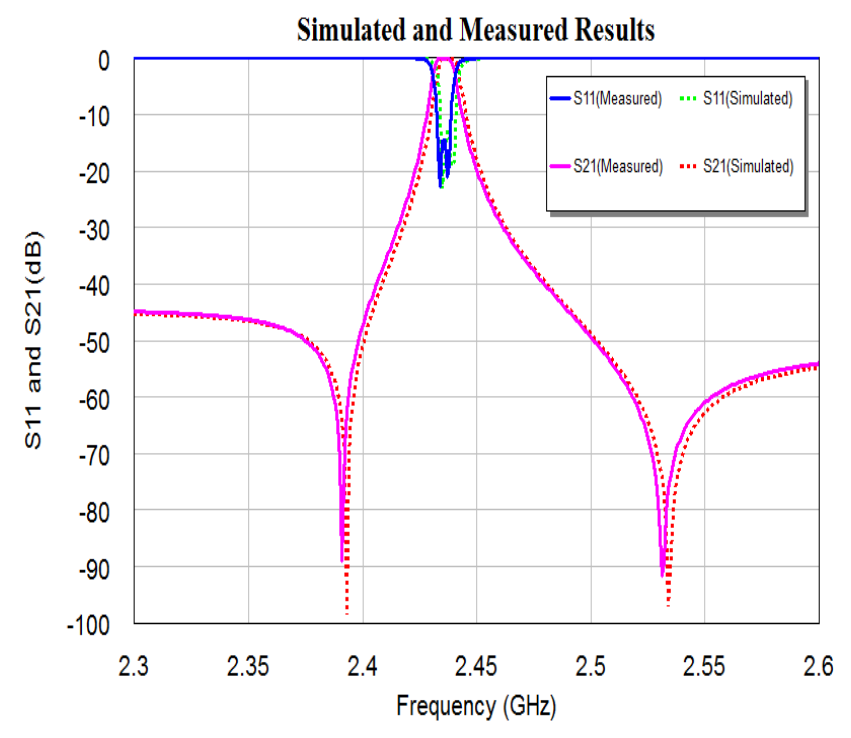

Figure 12. Simulated and measured frequency responses of designed quasi fractal BPF

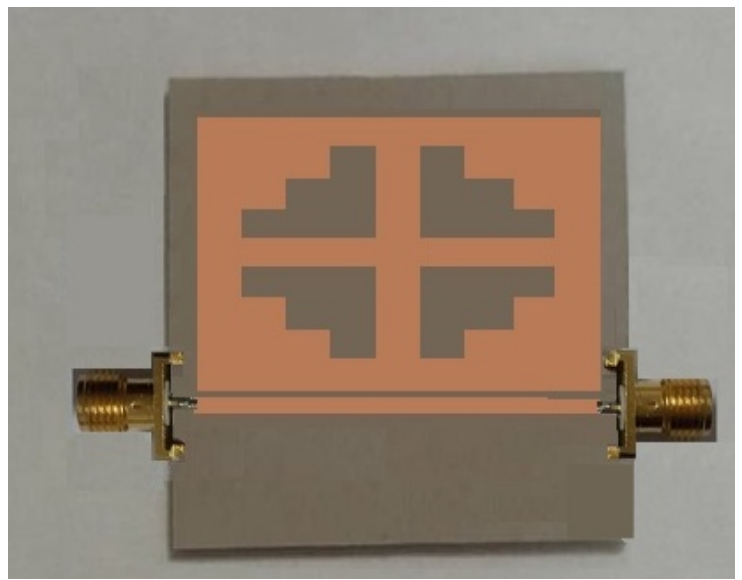

Figure 13. Photograph of the fabricated quasi fractal BSF

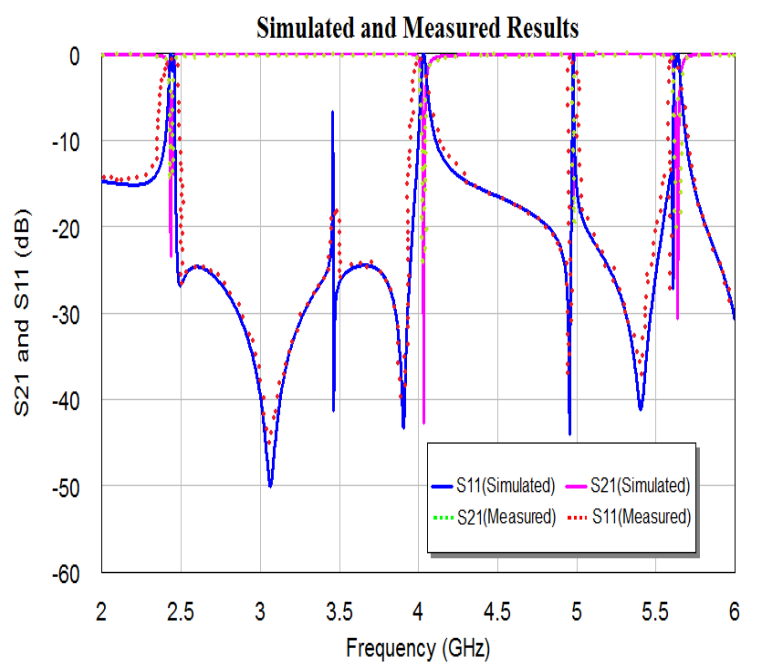

Figure 14. Simulated and measured frequency responses of designed quasi fractal BSF
The measured return loss magnitudes are better than 10 $\mathrm{dB}$ for quasi fractal $\mathrm{BPF}$ and $1 \mathrm{~dB}$ for all bands of quasi fractal BSF. On the other hand, the measured insertion loss magnitudes are better than $1 \mathrm{~dB}$ for quasi fractal BPF and $10 \mathrm{~dB}$ for all bands of quasi fractal BSF.As generalized in filter designs reported in $[16,33]$, the centre frequency of designed filters in this study can be changed according to intended band application by resizing the dimensions of quasi fractal resonator inversely proportional to the intended design frequency. Moreover, it is possible to optimize the electrical specifications of filter response by selecting $\mathrm{I} / \mathrm{O}$ feeder configurations with suitable feeder coupling gap, length and width. The bandwidth of filter responses can be adjustable by perturbation element as generalized in [18]. Changing the dimensions or the shape of slots can be used to adjust design frequency and change the bandwidth to some extent as generalized in filter designs reported in $[18,34]$.

\section{Conclusions}

In this study, microstrip BPF and multi band BSF filters have been presented. The proposed filters employ slotted patch microstrip resonator based on quasi fractal geometry, simulated by AWR12 software package. The proposed quasi fractal BPF is designed at centre frequency of $2.437 \mathrm{GHz}$, while quasi fractal multi band BSF is designed at band frequencies of $2.434,4.032,4.976$ and $5.638 \mathrm{GHz}$ $\mathrm{GHz}$ respectively. The presented quasi fractal BPF has extremely narrow fractional bandwidth of $0.38 \%$, which is theoretically extraordinary in microstrip filter design. On the other hand, quasi fractal BSF can be integrated within broadband wireless systems that are sensitive to permanent frequency interferences for ISM and C band wireless applications. The measured frequency responses match very well with the simulations with adequate performances.

\section{References}

[1] J.S. Hong and M.J. Lancaster(2001), Microstrip Filters for RF/Microwave Applications, John Wiley \& Sons.

[2] A. I. Zverev(1967), Handbook of Filter Synthesis. New York: John Wiley \& Sons.

[3] J. S. Hong and M. J. Lancaster(1996), Compact Microwave Elliptic Function Filter Using Novel Microstrip Meander Open-Loop Resonators, Electronics Letters, vol. 32, no. 6, pp. 563-564.

[4] Barra, M., Miniaturized Superconducting Planar Filters for Telecommunication Applications, PhD Thesis, University of Napoli, Italy, 2004.

[5] Y.S. Mezaal, H.T. Eyyuboglu and J.K. Ali, "'New Dual Band Dual-Mode Microstrip Patch Bandpass Filter Designs Based on Sierpinski Fractal Geometry", 
Proceeding of Advanced Computing and Communication Technologies, pp. 348-352, 2013.

[6] J. Romeu and J. Soler, "Generalized Sierpinski fractal multiband antenna," IEEE Transactions on Antennas and Propagation, vol. 49, no. 8, pp. 1237 - 1239, Aug. 2001.

[7] B. B. Mandelbrot, "The Fractal Geometry of Nature," New York, W. H. Freeman, 1983.

[8] O. I. Yordanov, I. Angelov, V.V. Konotop and I.V. Yurkevich "Prospects of Fractal Filters and Reflectors," in IEE Seventh International Conference on Antenna and Propagation ISCAP'91, pp. 698-700, York, UK, 1991.

[9] M. Barra. "Miniaturized Superconducting Planar Filters for Telecommunication Applications", $\mathrm{PhD}$ Thesis, University of Napoli, Italy, 2004.

[10] J.K. Ali, “A New Miniaturized Fractal Bandpass Filter Based on Dual-Mode Microstrip Square Ring Resonator," Proceedings of the $5^{\text {th }}$ International Multi-Conference on Signals, Systems and Devices, IEEE SSD '08, Amman, Jordan, July 20-23, 2008.

[11] Y.S. Mezaal, “A New Microstrip Bandpass Filter Design Based on Hilbert Fractal Geometry for Modern Wireless Communication Applications," International Journal on advanced Computing Techniques, IJACT, vol.1, no.2, pp.36-39, 2009.

[12] Ahmed A. Mahdi and Jabir S. Aziz, Miniaturized Koch Pre-Fractal Bandpass Filter, Journal of Mobile Communication 5 (5-6): 57-61, 2011.

[13]J. K. Ali, and Y. S. Mezaal, "A New Miniature Narrowband Microstrip Bandpass Filter Design Based on Peano Fractal Geometry," Iraqi Journal of Applied Physics, vol. 5, no. 4, pp. 3-9, 2009.

[14] J. K. Ali, and Y. S. Mezaal,"A new miniature fractalbased bandpass filter design with 2 nd harmonic suppression," Proceedings of 3rd IEEE International Symposium on Microwave, Antenna, Propagation and EMC Technologies for Wireless Communication, MAPE 2009.

[15] J. K. Ali, Hussam Alsaedi, Mohammed F. Hasan, and Hussain A. Hammas, "A Peano fractal-based dualmode microstrip bandpass filters for wireless communication systems." In PIERS Proceedings, pp. 888-892. 2012.

[16] Mezaal, Y. S., Jawad K. Ali, and H. T. Eyyuboglu. "Miniaturised microstrip bandpass filters based on Moore fractal geometry." International Journal of Electronics 102.8 (2015): 1306-1319.

[17] Y.S. Mezaal, H.T. Eyyuboglu and J.K. Ali (2014) Wide Bandpass and Narrow Bandstop Microstrip Filters Based on Hilbert Fractal Geometry: Design and Simulation Results, PLOS ONE DOI:10.1371/journal.pone.0115412

[18]Y.S. Mezaal and H.T. Eyyuboglu (2016) Investigation of New Microstrip Bandpass Filter Based on Patch Resonator with Geometrical Fractal Slot, Plos One 11(4) : e0152615. Doi:10.1371/ journal.pone.0152615.

[19] Yaqeen S. Mezaal, New Microstrip Quasi-Fractal Antenna: Design and Simulation Results, 2016 IEEE 36th International Conference on Electronics and Nanotechnology (ELNANO), Kiev,2016.

[20]Fattah Talaei, Mohammad N. Azarmanesh, Gholamreza Askari, and Hamid Mirmohammadsadeghi, "A novel dual-mode dual band bandpass filter using a quasi fractal structure," IEICE Electronics Express, Vol.7, No.3, 153-158, Feb. 2010.

[21] Ghatak, R., R. K. Mishra, D. R. Poddar, and A. Patnaik, "Multilayered complementary quasi-fractal Sierpinski patch antenna for wireless terminals," URSI XXVIII GA Poster Presentations Programme, 95, 2005.

[22] Jawad K. Ali, and A. S. A. Jalal, "A Miniaturized Multiband Minkowski-Like Pre-Fractal Patch Antenna for GPS and 3g IMT- 2000 Handsets", Asian Journal of Information Technology 6(5), pp. 584-588, 2007.

[23]F. Viani, " Dual-band Sierpinski Pre-Fractal Antenna for $2.4 \mathrm{GHz}$ - References $117 \mathrm{WLAN}$ and $800 \mathrm{MHz}-$ LTE Wireless Devices ", Progress In Electromagnetics Research C, PIERS, Vol. 35, pp. 6371, 2013.

[24] D. Zhang, G. C. Liang, C. F. Shih, M. E. Johansson, R. S. Withers, "Narrowband lumped element microstrip filter using capacitively loaded inductors", IEEE Transactions on Microwave Theory and Techniques, vol. 43, pp. 3030-3036, 1995.

[25] Ahmed, H.S., A.J. Salim, J.K. Ali, and N.N. Hussain. "A Compact Triple Band BSF Design Based on Minkowski Fractal Geometry." 18th IEEE Mediterranean Electrotechnical Conference, MELECON 2016, Limassol, Cyprus, April 2016.

[26] J.S. Hong, "Microstrip Filters for RF/Microwave Application," $2^{\text {nd }}$ edition, New York, Wiley, 2011.

[27] Saghlatoon H. and Neshati M. H. :Design Investigation of a Novel Bandpass Filter Using Trisection Open Loop Resonator. Progress In Electromagnetics Research Symposium Proceedings, Malaysia, 1203-1206, (2012).

[28] Chhabra A., Khanna R. and Kumar N.: A Novel Compact Band Pass Filter for $2.4 \mathrm{GHz}$ ISM Band Applications, Indian Journal of Science and Technology, vol. 9(47), DOI: 10.17485/ijst/2016/v9i47/106452, (2016).

[29] Arain S., Abassi Muhammad A. B., Nikolaou S. and Vryonides P. : A Square Ring Resonator Bandpass Filter With Asymmetrically Loaded Open Circuited Stubs. 5th IEEE International Conference on Modern Circuits and Systems Technologies, 2016.

[30] Weng, L. H., Guo, Y. C., Chen, X. Q. and Shi, X. W. 2008. Microstrip open loop resonator bandpass filter with DGS for WLAN application. Journal of Electromagnetic Waves and Applications, 22(No. 1415): 2045-2052.

[31] A. Griol, J. Marti, L. Sempere, "Microstrip multistage coupled ring bandpass filters using spur-line filters for harmonic suppression", Electrons Letters, vol. 37, no. 9, pp. 572-573, Apr. 2001. 
[32] Azzeddine Djaiz, and Tayeb A. Denidni, "A Design and Fabrication of a New Stacked Bandpass Filter Using Multilayer Microstrip Structure For Wireless Applications," IEEE Radio and Wireless Conference RAWCON, Boston, USA, pp. 317-320, Aug 2003.

[33] Mezaal YS, Eyyuboglu HT, Ali JK, New microstrip bandpass filter designs based on stepped impedance Hilbert fractal resonators. IETE Journal of Research, 2014; 60(3): 257-264.

[34] Y.S. Mezaal, H.T. Eyyuboglu: A new narrow band dual-mode microstrip slotted patch bandpass filter design based on fractal geometry. IEEE International Conference on Computing and Convergence Technology (ICCCT); 2012. 